\title{
Duodenal - pancreatic metastasis of primary prostate cancer: a case report
}

\begin{abstract}
Summary
Prostate cancer is one of the most frequent malignancies in men worldwide. Its metastatic behavior is well known, with gastrointestinal involvement being rare; however, as survival increases in patients with prostate cancer, metastases occur in unusual locations. We present the first case reported in the world literature with simultaneous duodenal and pancreatic involvement and fourth case with duodenal and pancreatic involvement separately. We report the case of an elderly patient with a history of prostate adenocarcinoma treated surgically 5 months ago, who presented with chronic abdominal pain, jaundice and choluria. The CT scan showed solid tumor in the head of the pancreas. Upper gastrointestinal endoscopy revealed an irregular ulcer in the duodenal bulb and extrinsic duodenal compression. The result of the pathological and immunohistochemical study revealed a metastatic prostate adenocarcinoma.
\end{abstract}

\section{Case presentation}

A 66-year-old male patient with a history of heavy cigarette consumption (46 packs - year) and prostate cancer (adenocarcinoma) treated with bilateral orchiectomy + radical prostatectomy was admitted to our hospital 5 months after surgery. He presented with 1-month history of progressively moderate non-irradiated epigastric pain associated with nausea, vomiting and hyporexia. Subsequently, jaundice and choluria were added. The physical examination at admission showed mild pallor, severe jaundice, a non-painful hard epigastric mass with a size of $8 \times 6 \mathrm{~cm}$ with poorly-defined edges, and dullness in flanks.

The laboratory tests showed hemoglobin $9.7 \mathrm{~g} / \mathrm{dL}$; creatinine 1.0 $\mathrm{mg} / \mathrm{dL}$; amilase $481 \mathrm{IU} / \mathrm{L}(<118)$; lipase $506 \mathrm{IU} / \mathrm{L}(<51)$; aspartate aminotransferase (AST) $68 \mathrm{IU} / \mathrm{L}(<34)$; alanine transaminase (ALT) $49 \mathrm{IU} / \mathrm{L}$ (10-49); total bilirrubin $21.2 \mathrm{IU} / \mathrm{L}(0.2-1.0)$ with conjugated fraction predominance $(17.8 \mathrm{mg} / \mathrm{dL})$; alkaline phosphatase $477 \mathrm{IU} / \mathrm{L}$ (45-129); albumin $3.1 \mathrm{~g} / \mathrm{dL}$ (3.5-5.5); total prostatic specific antigen
(PSA) $15.5 \mathrm{ng} / \mathrm{mL}$; Ca19.9 > $1200 \mathrm{IU} / \mathrm{L}$ (0-37); CEA 4.6 IU/mL (0-3); prothrombin time $(\mathrm{PT}) 15.8(<13)$; partial thromboplastin time $(\mathrm{TTP})$ $38.4(<37)$.

The ascitic fluid analysis showed pleocytosis (216 leukocytes/ $\mathrm{mm}^{3}$ ) with lympho-mononuclear predominance (91\%) and cytological analysis revealed malignant cells compatible with adenocarcinoma. The upper gastrointestinal endoscopy showed in the posterior wall of the duodenal bulb, a non-stenosing irregular ulcer that involved $50 \%$ of the circumference (biopsies were taken) and associated extrinsic compression that extended through the second duodenal portion also. Contrast-enhanced CT scan revealed inguinal lymphadenopathy and osteoblastic bone metastases in left coxal bone.

The patient underwent peritoneal catheter placement for ascites evacuation, which was removed 6 days later by negligible residual volume. Subsequently, an external biliary drainage catheter was placed at the level of the left hepatic duct. Patient showed favorable clinical course and was discharged with palliative care (Figure 1 \& Figure 2).
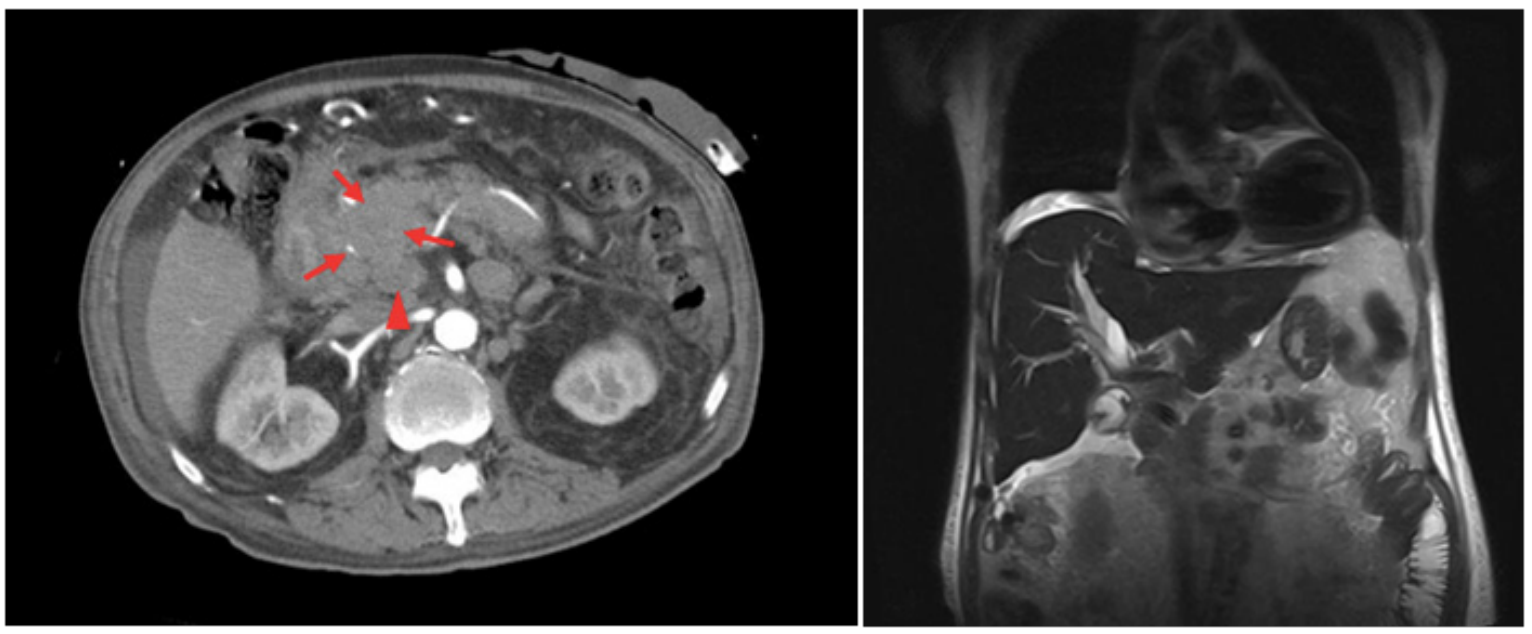

Figure IA: Contrast-enhanced CT scan: a hypodense heterogeneous poorly-defined mass of the head of pancreas (arrows) and retroperitoneal and mesenteric lymph nodes (arrowhead). Scarce peri-hepatic ascites. I B: MR cholangiography: Moderate intra and extrahepatic bile duct dilatation. 


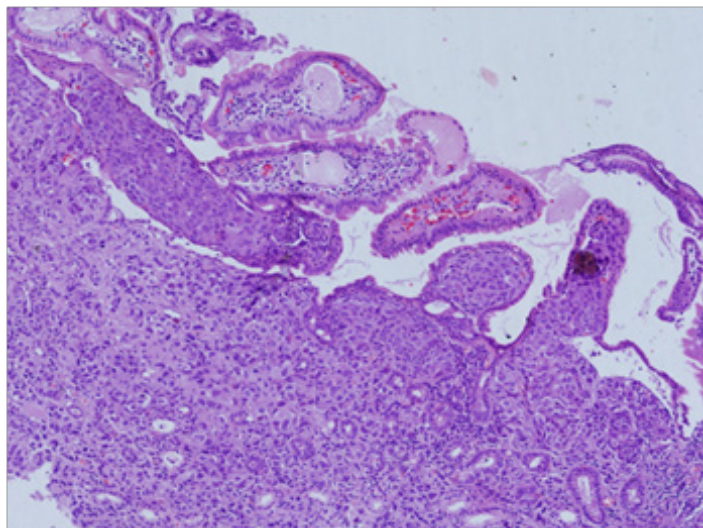

A

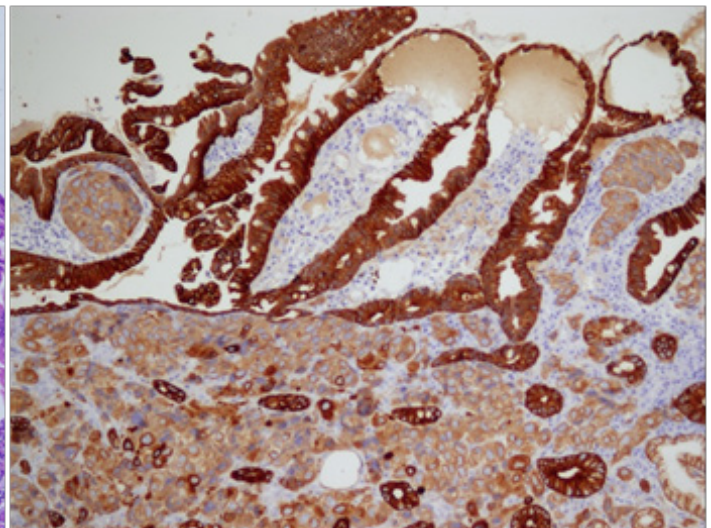

B

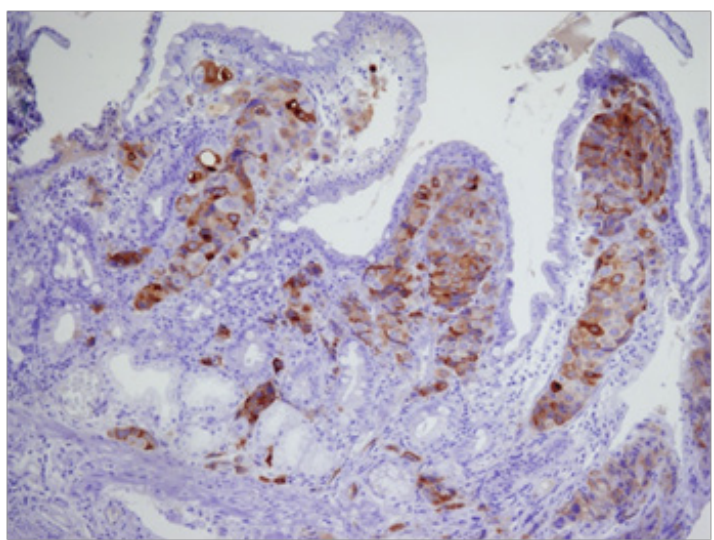

C

Figure 2 (A) Microphotograph showing poorly differentiated carcinoma with solid pattern in the lymphatic vessels and lamina propria of the duodenal mucosa (H-E, I00X). (B) Immunoreactive for Cytokeratin AI / A3 (200X) and (C) Prostate Specific Antigen (PSA) (200X).

\section{Discussion}

Prostate cancer is one of the most frequent malignancies in males worldwide. ${ }^{1}$ The most common metastases of this neoplasm are the regional lymph nodes, bones, lungs and liver, being the digestive affectation much more rare. ${ }^{2-4}$ On the other hand, the primary neoplasms that most frequently metastasize to the duodenum are renal cell carcinoma, malignant melanoma and breast cancer; ${ }^{2,5,6}$ while the pancreas is a rare location for other primary metastases, and when it occurs it is in the context of very advanced disease being the primary tumors most frequently reported renal (by far the most frequent), colorectal, gastric cancers among others..$^{7-9}$

According to a previous study, the lymphatic drainage of the prostate determines the location of the initial metastases, but subsequent metastatic extension probably originates from the sentinel lymph nodes. Likewise, metastases are more prevailing in patients with poorly differentiated tumors and in those who harbor capsule rupture. ${ }^{10}$ The metastatic involvement of prostate cancer to the gastrointestinal tract is rare and tends to be a local manifestation in which the rectum is affected. . $^{4,6}$

The symptoms that patients with duodenal or pancreatic metastases may present are non-specific, such as abdominal pain, abdominal distension, nausea, vomiting, jaundice or melena, the latter can be seen in the case of metastasis to the duodenum; however, it is considered that pancreatic metastases are asymptomatic in more than $50 \%$ of cases. ${ }^{3,4,9}$
As reviewed, this is the fourth case published worldwide of metastasis from a primary cancer of the prostate to the duodenum or to the pancreas (separately), and the first case reported in the literature of simultaneous metastasis to both organs of the digestive system by a prostate cancer. ${ }^{3,4,6,11}$ In general, metastases to the pancreas account for $1.4 \%$ of the total and occur once metastasis to other organs has already occurred. ${ }^{2,11}$ Because the evolution of prostate cancer is slow, it is more likely to find out unusual metastases like these ones as the survival in these patients increases., ${ }^{2,3}$

In conclusion, in the presence of a gastrointestinal tumor and a history of prostate cancer, the possibility of metastasis must be always considered (although digestive involvement is uncommon due to this cancer), especially in patients with a long history of disease and who had already metastases in other organs.

\section{Declaration of interest}

No conflicts of interest were declared.

\section{Acknowledgments}

None.

\section{References}

1. Siegel R, Ma J, Zou Z, et al. Cancer statistics, 2014. CA Cancer J Clin. 2014;64(1):9-29. 
2. Bubendorf L, Schöpfer A, Wagner U, et al. Metastatic patterns of prostate cancer: An autopsy study of 1,589 patients. Hum Pathol. 2000;31(5):578-583.

3. Kaswala DH, Patel N, Jadallah S, et al. Metastatic Prostate Cancer to the Duodenum: A Rare Case. J Family Med Prim Care. 2014;3(2):166-168.

4. Lee $\mathrm{S}$, Lee T, Yeh H, et al. An unusual case of metastatic small intestinal tumor due to prostate cancer. J Chin Med Assoc. 2009;72(5):271-274.

5. Kadakia S, Parker A, Canales L. Metastatic tumors to the upper gastrointestinal tract: endoscopic experience. Am J Gastroenterol. 1992;87(10):1418-1423.

6. Malhi-Chowla N, Wolfsen H, Menke D, et al. Prostate cancer metastasizing to the small bowel. J Clin Gastroenterol. 2001;32(5):439 -440 .
7. Merkle T, Boaz T, Kolokythas O, et al. Metastases to the pancreas. Br J Radiol. 1998;71(851):1208-1214.

8. Minni F, Casadei R, Perez B, et al. Pancreatic metastases: observations of three cases and review of the literature. Pancreatology. 2004;4(6):509520.

9. Zerbi A, Pecorelli N. Pancreatic metastases: An increasing clinical entity. World J Gastrointest Surg. 2010;2(8): 255-259.

10. Whitmore W. Procedings: The natural history of prostatic cancer. Cancer. 1973;32(5):1104-1112.

11. Jacob J, Chargari C, Bauduceau O, et al. Pancreatic metastasis from prostate cancer. Case reports in Medicine. 2010. 